\title{
Bronchoscopic Diagnosis of Solitary Pulmonary Nodules with the Use of NIR Spectroscopy
}

\author{
Votruba Jiri' ${ }^{1}$, Brůha Tomas ${ }^{1 *}$, Balaz Teodor ${ }^{2}$ \\ ${ }^{1}$ 1st Pulmonary Clinic, Charles University Prague, Praha, Czech Republic \\ ${ }^{2}$ University of Defense, Brno, Czech Republic \\ Email: ${ }^{*}$ enex@volny.cz
}

Received 29 September 2015; accepted 19 October 2015; published 22 October 2015

Copyright (C) 2015 by authors and Scientific Research Publishing Inc.

This work is licensed under the Creative Commons Attribution International License (CC BY). http://creativecommons.org/licenses/by/4.0/

(c) () Open Access

\section{Abstract}

Background: Recently, SPN has become a much more frequently encountered issue in bronchology. Efficient and reliable guidance method for SPN morphological proof is highly needed. Objectives: The aim of study was to compare the diagnostic values of NIR (near infrared) spectroscopy with EBUS for SPN diagnostic. Fluoroscopic guidance with TBB and needle biopsy were done in all patients. Methods: In our study, we used two types of monitoring systems. Fluoroscopic guidance was combined with either a radial EBUS or a NIR spectroscopy probe for tissue confirmation. 139 male and 71 female patients, having a medial age of 68 years with CT/PET findings of metabolically active SPN were examined between $2 / 2010$ and $2 / 2013$. We designed an instrument for measurement of the penetration of the NIR through lung tissue. Indicating and source fibers were navigated towards the SPN. An EBUS radial probe was used, during fluoroscopic navigation. Results: The statistical analysis of the results obtained showed a comparative specificity and sensitivity of the NIR spectroscopy, with radial EBUS. Conclusions: NIR spectroscopy produced similar efficacies as the radial EBUS. However, the number of positive biopsies was more dependent upon the ability to direct the confirmatory device to the SPN during fluoroscopic guidance than on the type of the device.

\section{Keywords}

Solitary Pulmonary Nodule, Near Infrared Spectroscopy, Endobronchial Ultrasound, Fluoroscopy, Biopsy

\footnotetext{
${ }^{*}$ Corresponding author.
}

How to cite this paper: Jiri, V., Tomas, B. and Teodor, B. (2015) Bronchoscopic Diagnosis of Solitary Pulmonary Nodules with the Use of NIR Spectroscopy. Advances in Chemical Engineering and Science, 5, 490-498. 


\section{Introduction}

A solitary pulmonary nodule (SPN) is a single abnormality in the lung on a chest X-ray that is smaller than $3 \mathrm{~cm}$, surrounded by normal lung tissue, and is not associated with any other abnormality in the lung.

Recently, SPN has more often been encountered due to the widespread use of CT for diagnostic and screening purposes. According to a recent study [1], incidental findings, especially of pulmonary nodules, are as prevalent as $18 \%$ in the population of older healthy adults examined by cardiac MDCT, performed to assess coronary artery calcification. In eight lung cancer screening studies there was an $8 \%-51 \%$ prevalence of at least one nodule, as well as a $1.2 \%$ - $12 \%$ prevalence of malignancy in those patients with nodules [2].

Due to ACCP Evidence-Based Clinical Practice Guidelines, neither the "time-honored" rule of 2-year radiographic stability, nor the radiological characteristics of SPN are reliable in distinguishing malignant from benign lesions [3].

There has been a similar accuracy between the imaging modalities (dynamic CT, Dynamic NMR, FDG-PET, and Tc-dep SPECT) in distinguishing of malignant from benign SPN in a recent meta-analytic comparison [2]; the sensitivity was $93 \%-95 \%$, and the specificity was $76 \%-82 \%$. However, it is important to be both accurate and efficient in the diagnostic evaluation of SPN, as the prompt resection of malignant tumors can be lifesaving (in patients with resected malignant nodules, survival may be as high as 80 percent, five years on), but it is also prudent to avoid surgery in the case of benign disease. To date, the optimal management strategy for SNP remains unclear.

In order to obtain histological or cytological proofs of the SPN origin, we can employ several bronchologic and invasive radiology methods.

Transthoracic needle aspiration with CT guidance is a well established method, which has a diagnostic accuracy of about $90 \%$ with SPN larger than $2 \mathrm{~cm}$ in diameter. The accuracy decreases (to 60\% - 80\%) in nodules that are smaller than $2 \mathrm{~cm}$ in diameter. The risk of pneumothorax precludes its use in respiratory unfit patients.

Until recently, bronchoscopy used to have a limited role in the evaluation of SPN. Transbronchial biopsy under fluoroscopic guidance has approximately a 60\% diagnostic success for peripheral, endobronchially unreachable nodules, and the hit rate drops further (to 25\% - 30\%) for those SPNs smaller than $2 \mathrm{~cm}$ [3].

However, the list of bronchological methods used for the diagnosis of SPN has recently been enlarged; we can now employ: ultrathin bronchoscopy, endobronchial ultrasound (EBUS), electromagnetic navigation, and virtual bronchoscopic navigation. According to the literature, radial EBUS guided transbronchial biopsy has obtained a 67\% success rate in the diagnosis SPN smaller than 2 centimeters [4] [5].

In our work, we utilized NIR spectroscopy of penetrated light, in order to enhance the diagnostic value of the fluoroscopically guided transbronchial biopsy technique.

Near-infrared radiation (NIR) is defined as light with wavelengths from 700 to $2500 \mathrm{~nm}$. Such radiation passes fairly easily through several centimeters of biological samples, due to its relatively low absorbance [6]. Its interaction with biological tissue is illustrated in Figure 1. The radiant flux coming to the tissue surface is subsequently scattered in the tissue, while another part is absorbed in the tissue, part is reflected back, and a portion is transmitted through it.

The ratio between the transmitted radiant flux and total radial flux coming to the surface is defined as the transmittance coefficient

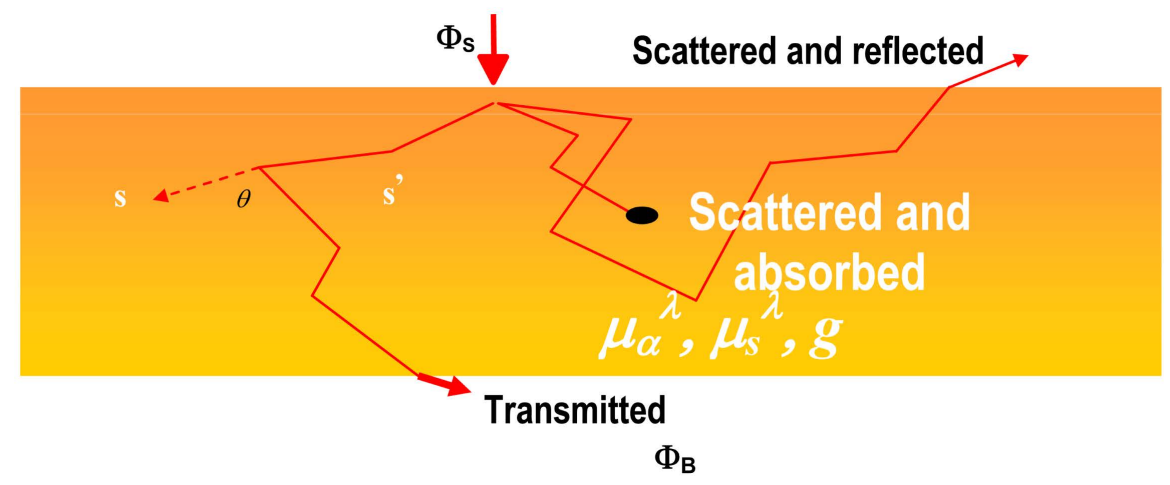

Figure1. Interaction of photons with biological tissues. 


$$
\tau=\Phi_{\mathrm{B}} / \Phi_{\mathrm{S}}
$$

or as the spectral transmittance coefficient

$$
\tau_{\lambda}=\Phi_{\mathrm{B} \lambda} / \Phi_{\mathrm{S} \lambda}
$$

respectively, for a certain wavelength.

As can be seen, this transmittance coefficient depends on the optical characteristics of the tissue being investigated, represented by the absorption coefficient $\mu_{a}^{\lambda}$, scattering coefficient $\mu_{s}^{\lambda}$, and the index of refraction $g$, as well as on the anisotropy, with respect to the isotropy properties of the tissue. It can be assumed that the aforementioned optical characteristics are in strong correlation with the morphological characteristics of the tissue.

Therefore our question was: can NIR light penetrating through the parenchyma bring forth the information about the presence of a SPN; and secondarily, is it able to give us some information about its morphological properties? The scattering of light in a tissue is caused by inhomogeneities, such as cell membranes or intracellular structures. The scattering arises due to the relative refractive index mismatches at the boundaries between two such media or structures (e.g. between the extracellular fluid and the cell membrane).

Light scattering and absorption can provide information both about the tissue structure and on the chromophore content; these are features that can be used to distinguish between normal tissues, malignant lesions, and other pathologies. This is why we speculated that NIR light penetrating normal lung parenchyma will have different spectral and intensity characteristics than will the light from the same source when penetrating a solitary pulmonary nodule.

For that purpose, we designed and carried out a number of ex-vivo studies on tissue models and cadavers, which confirmed the rationale behind this idea.

Due to the fact that human tissues are comprised of a physical medium characterized by both prominent absorbance and dispersion of penetrating light, the spectral transmittance coefficient of the measured flux $\tau_{\lambda}$ reached values on the order of up to $10^{-4}$ [7]. It is for this reason that in the selected spectral band we were able to analyze the standardized scanned spectral curve of the transmission coefficient, with regard to its maximal measured value by use of the formula:

$$
\tau_{\lambda_{n}}=\frac{\tau_{\lambda}}{\tau_{\max \left\langle\lambda_{\min } ; \lambda_{\max }\right\rangle}}
$$

This method enables us to compare the results that have been acquired from sources of different spectral emission curves [6].

That is why we carried out a randomized prospective comparative study, in order to discriminate the efficiency of the two techniques: transbronchial biopsy under fluoroscopic guidance, and NIR spectroscopy guided transbronchial biopsy.

\section{Materials and Methods}

This Work Was Conducted under the Clinical Protocol Thermo/03/07, and Was Approved by the Local Ethics Committee of Na Homolce Hospital's IRB.

\subsection{Study Design}

The patient population consisted of 210 patients with CT/PET findings of metabolically active solitary pulmonary nodes from 1.1 to $3 \mathrm{~cm}$ in diameter. They consisted of 139 male and 71 female patients, with a median age of 68 years. Out of this population 82\% were smokers, with a homogenous distribution in both groups. The patients were randomized into the groups: fluoroscopic + EBUS TBB, and the NIR confirmed fluoroscopic TBB, in a fashion leading to a ratio of 1:1. The fluoroscopic + EBUS TBB group vindicated the standard TBB process, as described elsewhere [8]. The other group had confirmatory NIR spectroscopy, following fluoroscopic navigation using the method of real-time measurements from target areas. There was never more than one lesion biopsied in any one patient. Distribution of the disease types in cohorts of the patients is presented in Table 1.

The principle of a technical solution of the spectroscopic probe and NIR spectroscope is shown in Figure 2. 


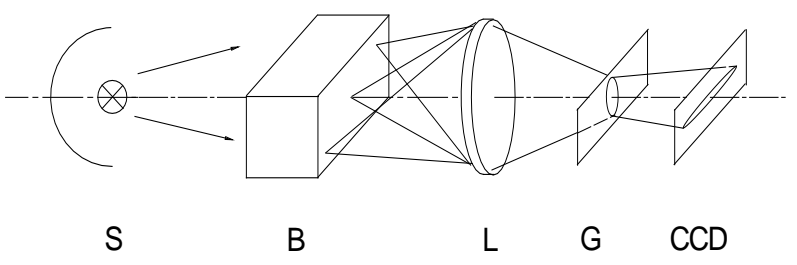

Figure 2. Adjustment of spectral transmission measurements of tissue. $\mathrm{S}$ is the source of radiation, $\mathrm{B}$ is the tissue measured, $\mathrm{L}$ is the optical focusing system, $\mathrm{G}$ is the diffraction grating, CCD is the CCD line detector.

Table 1. Distribution of disease types in our cohort of patients.

\begin{tabular}{lcc}
\hline \multicolumn{1}{c}{ Total number of positive findings } & $\mathbf{1 5 6}$ \\
\hline NSCL & Adenoca & $\mathbf{8 3}$ \\
& Epidermoid & 55 \\
& BAC & 15 \\
& Large cell carcinoma & 6 \\
SCLC & & 7 \\
Sarcoidosis & 16 \\
Tuberculoma & 21 \\
Pulmonary embolism & $\mathbf{1 3}$ \\
Inflammatory changes & $\mathbf{2}$ \\
Nonprimary tumor generalized to the lung & $\mathbf{1 4}$ \\
\hline
\end{tabular}

Spectral measurements were carried out using an AVANTES AVS-PC 2000, Plug-in Spectrometers PC2E1260 Master. The spectrometer has a linear CCD detector with 2048 pixels. Its working range is from 200 to $1100 \mathrm{~nm}$. The integration time for the whole spectrum is from $3 \mathrm{~ms}$ to $60 \mathrm{~s}$. The spectral grating UV/VIS/NIR\#13 works in the spectral band from 300 to $1100 \mathrm{~nm}$, and it has 7 lines $/ \mathrm{mm}$. The calibration curves of the spectrometer are presented in Figure 3.

\subsection{Methods}

We designed a simple instrument for the measurement of the penetrated NIR light through the lung tissue. It consists of two fibers, $1 \mathrm{~mm}$ in diameter, contained in one bundle covered with an insulation sleeve. One of the fibers is the detector, while the other is the source fiber. The indicator fiber is $0.3 \mathrm{~cm}$ longer than source fiber, and it is separately covered with insulation up to its end. The end is cut at the angle of 60 degrees and is titanium coated in order to facilitate NIR light transmission toward the detector fiber. The detector fiber is connected to the NIR spectroscope and the source fiber to the NIR source, the parameters of which are shown at Figure 4. The scheme of our experimental measuring system is presented in Figure 5. Measurements of pathological lung tissues showed a characteristic peak around $673 \mathrm{~nm}$, while during normal tissue spectroscopy this peak was shifted toward longer wavelengths. With the aid of those finding, we utilized the endobronchial measurement of the NIR transmittance spectra in an attempt to improve the sensitivity of a transbronchial biopsy under fluoroscopic guidance.

Data from the NIR spectroscopy has been displayed and interpreted in real-time during the bronchoscopic examination. Whenever a spectral pattern and intensity change that were not characteristic of normal lung parenchyma appeared on the spectroscopic monitor (Figure 6(a) and Figure 6(b)) we changed the spectroscopic probe to a flexible forceps, and performed the biopsy under fluoroscopic guidance from the same area as the area of the spectroscopic measurement. 


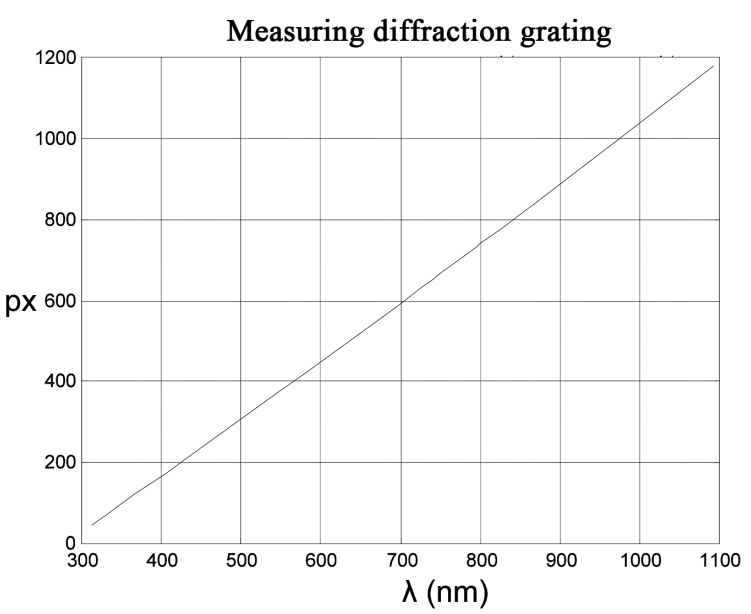

(a)

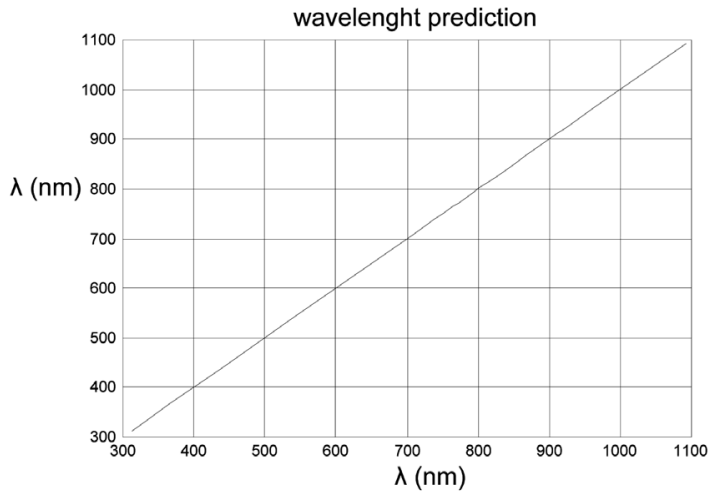

(b)

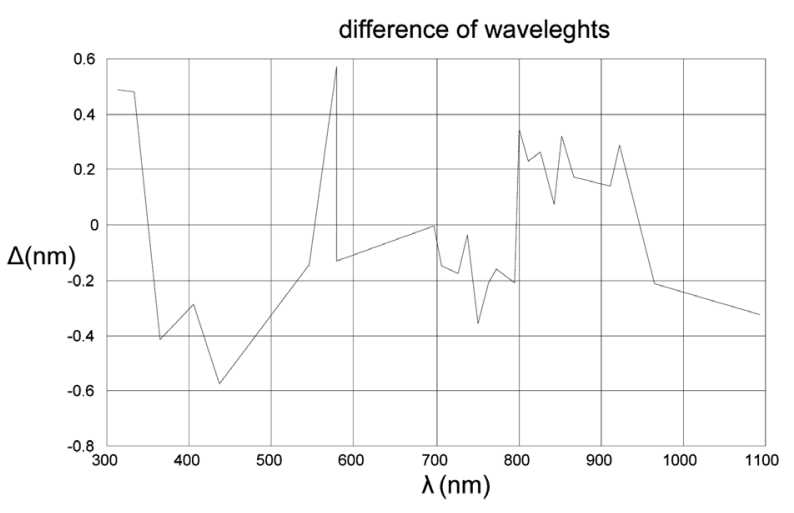

(c)

Figure 3. Calibration curves of NIR spectrometer. (a) Calibration curve of the measuring diffraction grating of the AVANTES AVS-PC 2000 spectrometer; (b) Calibration curve of measured wavelength prediction; (c) Difference of real and measured wavelengths by the spectrometer.

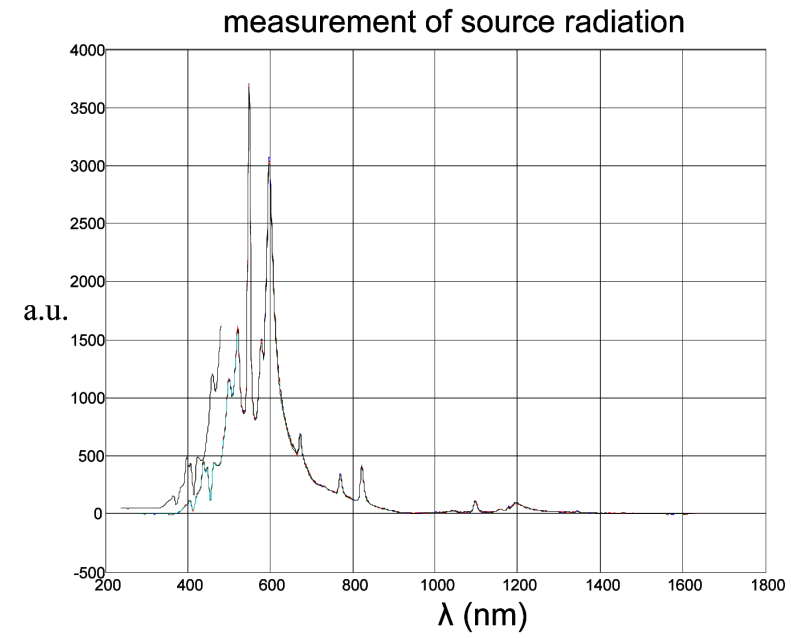

(a)

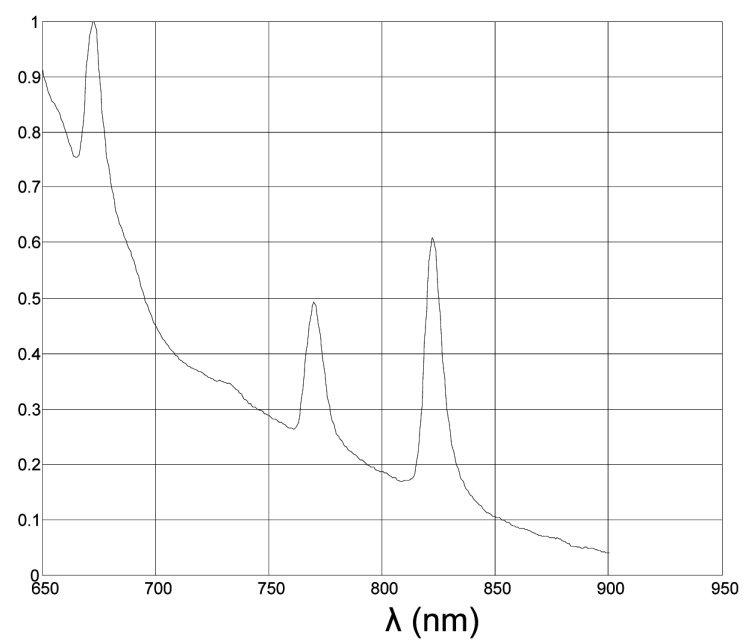

(b)

Figure 4. Behavior of the SÓLARC Light ELS.24DC light source-the mean spectral curve of the light source utilized for the measurement evaluation. (a) Spectral radiation of the source; (b) Standardized spectral curve for wavelength interval. $\Delta \lambda=\langle 650 ; 900\rangle \mathrm{nm}$. 


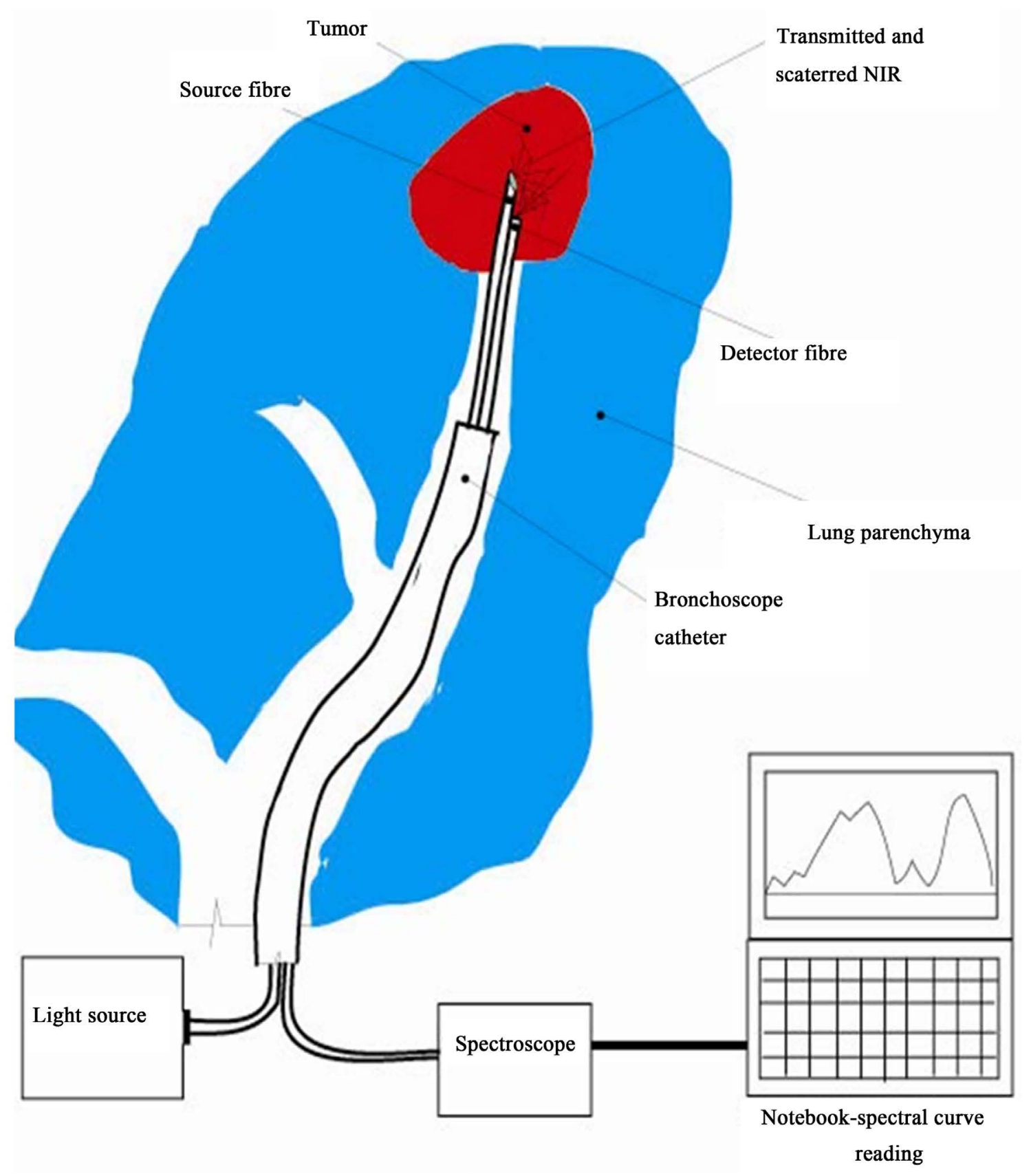

Figure 5. Scheme of experimental measuring system.

\subsection{Analysis}

In order to quantify the results of the NIR spectroscopy, we calculated the intensity ratio of $\mathrm{I}_{673 \mathrm{~nm}} / 694 \mathrm{~nm}$ for each spectral curve of the normalized transmittance (see Figure 6(a) and Figure 6(b)). Consequently, we applied the statistical comparative analysis (unpaired Students $t$-test for significance of the difference of two means) between sets of intensity ratio values for both pathological tissues and normal tissues, as resulted from biopsies of the Sensitivity, respectively TBB hit rate were determined for the NIR navigation and the EBUS navigation, respectively.

\section{Results}

Of the 210 eligible patients examined from 2/2010 to 2/2013, evaluable biopsies were obtained. Table 2 summarizes 


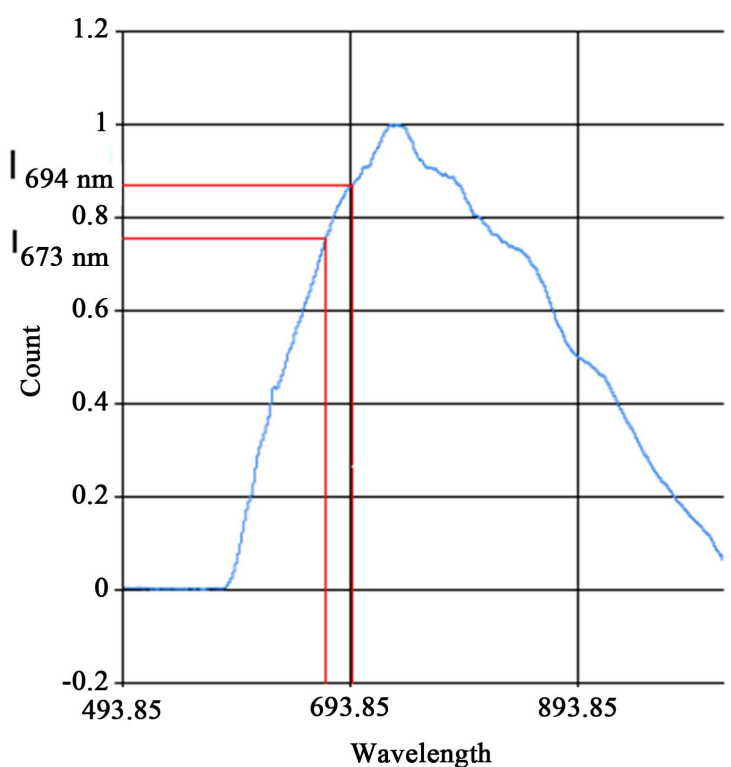

- Count

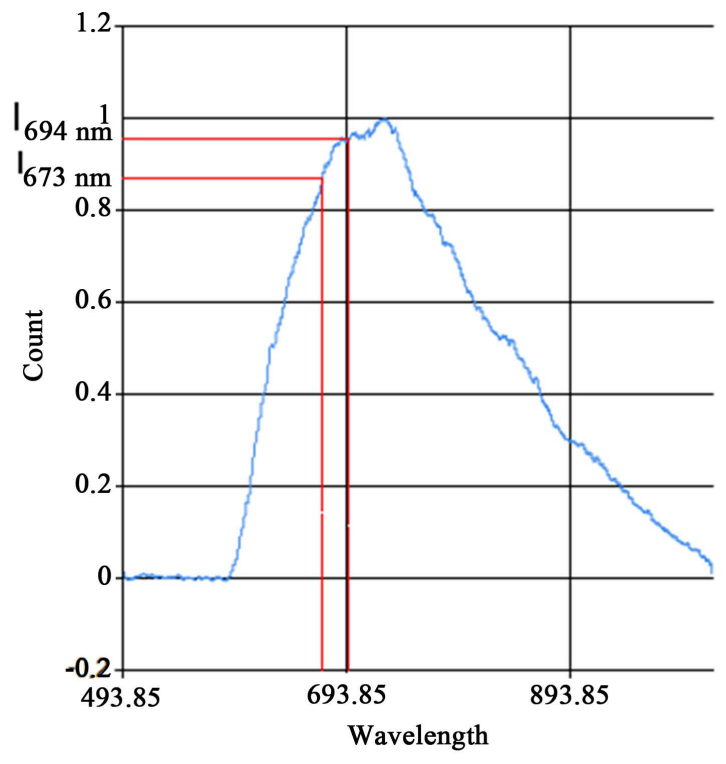

- Count

(a)
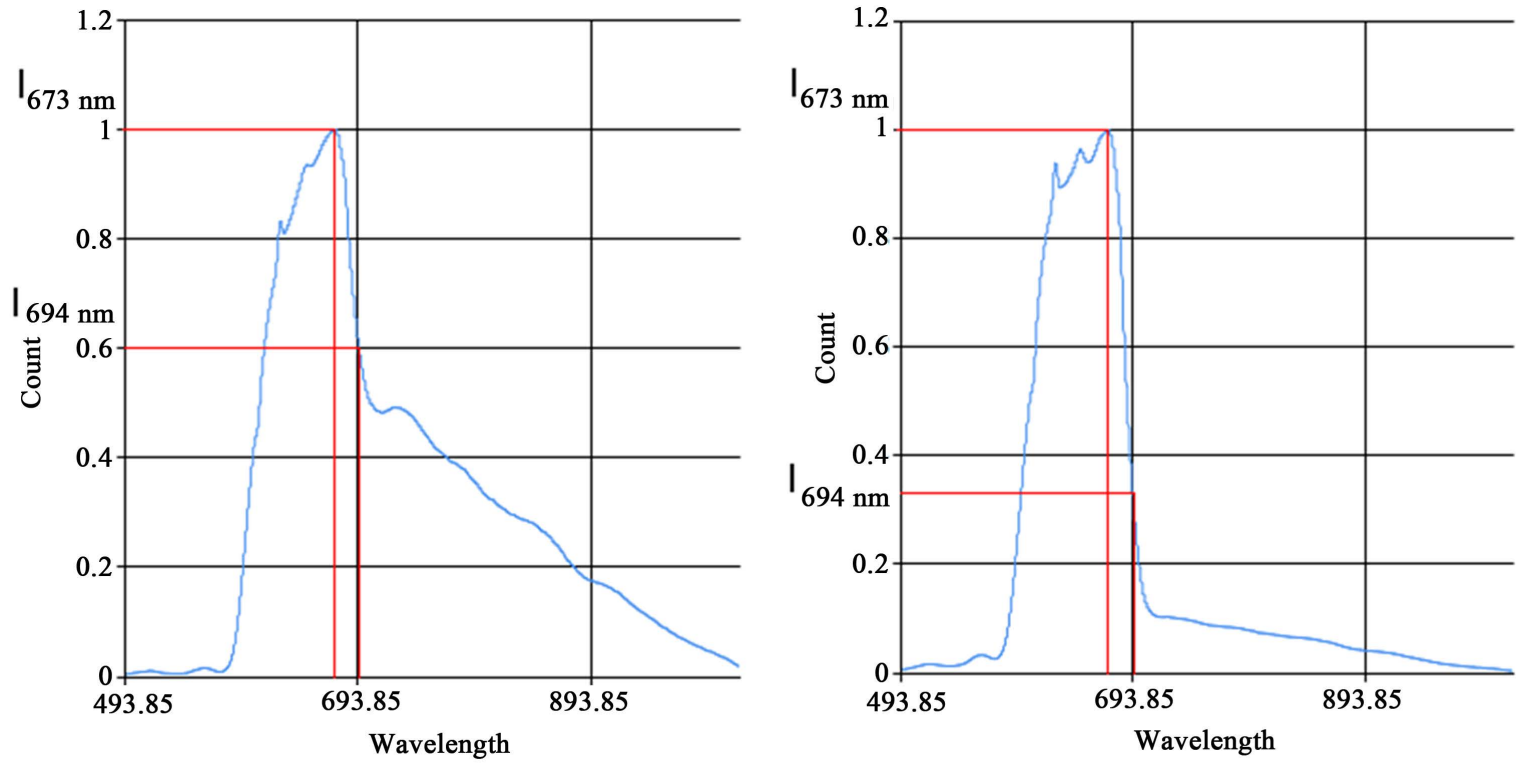

- Count

(b)

Figure 6. (a) Samples of spectral curves of normalized transmittance in normal areas, with reading of intensity values at two selected wavelengths; (b) Samples of spectral curves of normalized transmittance at pathological areas, with reading of the intensity values at two selected wavelengths.

Table 2. Summary of the results of histologic examination.

\begin{tabular}{ccccc}
\hline Method & $\begin{array}{c}\text { No. } \\
\text { of lesions }\end{array}$ & $\begin{array}{c}\text { No. of positive } \\
\text { histologies in all lesions }\end{array}$ & $\begin{array}{c}\text { No. of negative } \\
\text { histologies in all lesions }\end{array}$ & $\begin{array}{c}\text { Sensitivity } \\
\text { rate (\%) }\end{array}$ \\
\hline FLUORO + NIR & 106 & 82 & 24 & 0.77 \\
FLUORO + EBUS & 104 & 74 & 30 & 0.71 \\
\hline
\end{tabular}


the results of the histology with both of the navigational methods.

Table 3 presents the results of the statistical analysis. It was shown that a 95\% CI for the intensity ratio of pathological tissue represents significantly higher values than the $95 \%$ CI of normal tissue; the mean difference of the ratios of the values between the pathological and normal tissues is 1.51. In the NIR navigation, group 2 values of the intensity ratio from the pathological location set lie within the $95 \% \mathrm{CI}$ of the intensity ratio values for normal locations, and the 2 values of the intensity ratio from the normal location set lie within the $95 \%$ CI of the ratio values for pathological locations. False negative cases were probably due to the mis-positioning of the measuring probe, while false positive cases were likely due to incorrect spectroscopic readings. In the EBUS group, it was impossible to visualize the lesion in 30 cases, and there were no false positive readings. The corresponding characteristic values for both methods are presented in Table 2 and Table 4 [4] [5].

The level of significance, reached after testing the hypothesis of inequality of the mean for the pathological intensity ratio values and normal intensity ratio values, was 0.0001 . Also based on the results of this statistical analysis, we can state that the cut-off value of the intensity ratio $\mathrm{I}_{673 \mathrm{~nm}} / \mathrm{I}_{694 \mathrm{~mm}}$ for the diagnosis of pathological tissue at 1.3 , while that for the diagnosis of normal tissue is 0.95 .

\section{Discussion}

NIR light is used routinely in numerous applications in modern medicine [9]. Our approach differed in using penetrated, instead of reflected, NIR radiation. We took into consideration the possibility of interference of hemoglobin redox status with penetrating radiation. However, in the spectral range of $680-720 \mathrm{~nm}$ there is very limited absorption of NIR radiation in both oxydated and reduced hemoglobins. Hence, it appears that this method might bring to us new qualities in the endoscopic examination of solitary pulmonary nodules. Its first notable advantage is very low cost. Another possible advantage is the opportunity to miniaturize the device (as the distal part of the device consists from only 2 fibers it can be miniaturized incomparably easier than radial EBUS and possibly incorporated into single use instruments).

From our first data, we concluded that the use of NIR spectroscopy for ideal biopsy area confirmation has been useful in increasing the sensitivity of fluoroscopically guided TBB and comparable to EBUS navigated TBB. Such a device, or its modification, could easily be included e.g. into an examination by electromagnetic navigation or single use, low cost catheters.

The main disadvantage of the method appears to be its limitation to point monitoring, and hence the necessity of a trial and error method in the guidance. This shortcoming is the same one as in the case of radial endoscopic ultrasound.

\section{Acknowledgements}

This work originated with financial support from the industrial research project of the Ministry of Industry and Trade of the Czech Republic-project code FR-TI4/765: "Research and development of technologies and

Table 3. Statistical results for NIR spectral change.

\begin{tabular}{ccccccc}
\hline & $\mathbf{N}$ & SD & Mean & CI 95\% L & CI 95\% U & $\boldsymbol{p} \leq \quad$ \\
\hline $\mathbf{I}_{673 \mathrm{~nm}} / \mathbf{I}_{\mathbf{6 9 4 m m}}$ pathological & 82 & 1.11 & 2.29 & 1.31 & 3.05 & \\
$\mathbf{I}_{\mathbf{6 7 3 n m}} / \mathbf{I}_{694 \mathrm{~nm}}$ normal & 24 & 0.28 & 0.78 & 0.64 & 0.91 & 0.0001 \\
\hline
\end{tabular}

Table 4. Results for lessions smaller than $2 \mathrm{~cm}$ [7].

\begin{tabular}{ccccc}
\hline Method & $\begin{array}{c}\text { No. } \\
\text { of lesions } \\
\text { smaller } \\
\text { than } 2 \mathrm{~cm}\end{array}$ & $\begin{array}{c}\text { No. of positive } \\
\text { histologies in } \\
\text { lesions smaller } \\
\text { than } 2 \mathrm{~cm}\end{array}$ & $\begin{array}{c}\text { No. of negative } \\
\text { histologies in } \\
\text { Lesions }\end{array}$ & $\begin{array}{c}\text { Sensitivity } \\
\text { smaller than } 2 \mathrm{~cm}\end{array}$ \\
\hline FLUORO + NIR & 38 & 25 & 13 & 0.66 \\
FLUORO + EBUS & 45 & 33 & 12 & 6.73 \\
\hline
\end{tabular}


methods for the early diagnosis of lung cancer using NIR spectroscopy”, the Ministry of Health of the Czech Republic (project No. NT13259) and the Czech Science Foundation (project No. P208/11/0105).

\section{References}

[1] Burt, J.R., Iribarren, C., Fair, J.M., et al. (2008) Incidental Findings on Cardiac Multidetector Row Computed Tomography among Healthy Older Adults: Prevalence and Clinical Correlates. Archives of Internal Medicine, 168, 756-761. http://dx.doi.org/10.1001/archinte.168.7.756

[2] Ost, D., Fein, A.M. and Feinsilver, S.H. (2003) Clinical Practice: The Solitary Pulmonary Nodule. The New England Journal of Medicine, 348, 2535-2542. http://dx.doi.org/10.1056/NEJMcp012290

[3] Wahidi, M.M., Govert, J.A., Goudar, R.K., Gould, M.K. and McCrory, D.C. (2007) Evidence for the Treatment of Patients with Pulmonary Nodules: When Is It Lung Cancer ACCP Evidence-Based Clinical Practice Guidelines (2nd Edition). CHEST, 132, 94S-107S.

[4] Cronin, P., Dwamena, B.A., Kelly, A.M. and Carlos, R.C. (2008) Solitary Pulmonary Nodules: Meta-Analytic Comparison of Cross-Sectional Imaging Modalities for Diagnosis of Malignancy. Radiology, 246, 772-782.

[5] Eberhardt, R., Ernst, A. and Herth, F.J.F. (2009) Ultrasound-Guided Transbronchial Biopsies of Solitary Pulmonary Nodules Less than 20 mm. ERJ, 34, 1284-1287. http://dx.doi.org/10.1183/09031936.00166708

[6] Ntziachristos, V., Ripoll, J. and Weissleder, R. (2002) Would Near-Infrared Fluorescence Signals Propagate through Large Human Organs for Clinical Studies? Optics Letters, 27, 333. http://dx.doi.org/10.1364/OL.27.000333

[7] Pogue, B.W., Patterson, M.S., et al. (2006) Review of Tissue Simulating Phantoms for Optical Spectroscopy, Imaging and Dosimetry. Journal of Biomedical Optics, 11, 041102. http://dx.doi.org/10.1117/1.2335429

[8] Prakash, U.B.S. (2005) Bronchoscopy. In: Mason, R.J., Murray, J.F., Broaddus, V.C. and Nadel, J.A., Eds., Murray \& Nadel's Textbook of Respiratory Medicine, 4th Edition, Saunders Elsevier, Philadelphia, Ch 22.

[9] Wolf, M., Ferrari, M. and Quaresima, V. (2007) Progress of Near-Infrared Spectroscopy and Topography for Brain and Muscle Clinical Applications. Journal of Biomedical Optics, 12, 062104. 